\title{
An Improved Method for Heteronuclear Chemical Shift Correlation by Two-Dimensional NMR
}

Since the use of two-dimensional NMR for correlating the spectra of coupled heteronuclei was first proposed in $1977(1)$, a considerable number of papers have appeared describing different techniques for heteronuclear correlation (2-8). The most widely used method $(5,9-12)$ produces a two-dimensional spectrum in which one signal appears for each directly bonded carbon-hydrogen pair in a molecule. The $f_{1}$ domain of the spectrum is governed by the proton resonance frequencies and the $f_{2}$ by the carbon-13, so that the correlation signal for a given $\mathrm{CH}_{n}$ group appears at frequency coordinates which are just the proton chemical shift in $f_{1}$, and the carbon-13 chemical shift in $f_{2}$. Heteronuclear couplings are absent from both frequency domains, although proton-proton scalar coupling structure remains in $f_{1}$. Such experiments are relatively simple to perform, and show good sensitivity; data for a typical shift correlation two-dimensional spectrum may be obtained in about 10 times the time required to produce a good protondecoupled carbon-13 spectrum.

A number of chemical applications of such shift correlation experiments have been published $(9-11)$, all using the basic pulse sequence of Ref. (5). This sequence generates proton-decoupled carbon-13 free-induction decays which are modulated as a function of $t_{1}$ by the frequency difference between the proton chemical shift and the proton transmitter frequency. Since the signals are amplitude modulated with respect to $t_{1}$, it is not possible to distinguish between positive and negative $f_{1}$ frequencies (13), so that to avoid ambiguity it is necessary to position the proton transmitter either to low field or to high field of the proton spectrum. This is unfortunate in that much of the proton transmitter power is thus wasted, which can lead to severe heating problems with ionic samples if the same proton frequency is used for pulses and for decoupling, as is usually the case. A second problem is that twice as many $t_{1}$ samples are needed to digitize the $f_{1}$ domain of the spectrum if the proton transmitter lies to one side of the proton spectrum. Both from the point of view of optimum decoupling and from that of minimizing data storage needs and complexity of data processing, it would be desirable to be able to place the proton transmitter in the middle of the proton spectrum.

This communication suggests the use of phase cycling of the proton and carbon-13 transmitter pulses, similar to that recently introduced in homonuclear correlation experiments (14-16). This converts amplitude modulation as a function of $t_{1}$ into phase modulation, allowing positive and negative $f_{1}$ frequencies to be distinguished. Thus in the usual experiment (5), examination of $f_{2}$ spectra for successive $t_{1}$ values would show the amplitudes of individual signals oscillating while their phases remained fixed. The proposed modification would lead to $f_{2}$ spectra with signals oscillating between absorption and dispersion mode while 

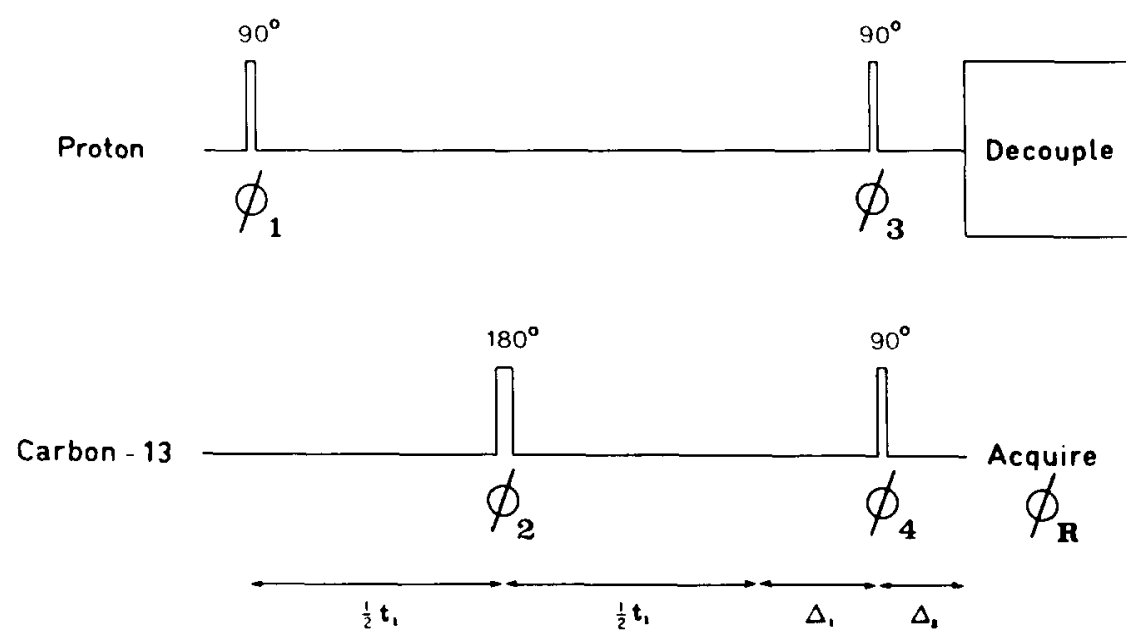

Fig. 1. Pulse sequence used for measuring two-dimensional chemical shift correlation spectra; the phases $\phi_{1-4}$ of the various pulses and of the detection $\phi_{\mathrm{R}}$ are cycled on successive transients during signal averaging, according to the scheme summarized in Table 1.

keeping nearly the same absolute intensity, the sense of the phase rotation being determined by the sign of the offset of the appropriate proton resonance from the proton transmitter frequency. The pulse sequence of Fig. 1 is retained (5), but the receiver and the proton and carbon- 13 transmitter phases are changed in cyclic fashion on successive transients during time averaging, as summarized in Table 1 . The resultant technique is thus significantly less complex than the data manipulation of the "hypercomplex Fourier transformation" method proposed by Müller and Ernst (7).

The mechanism of chemical shift correlation experiments has been extensively discussed in the literature $(1-3,6,7,9,11)$ and will not be described here. A single transient obtained using the pulse sequence of Fig. 1 ungarnished with phase shifts will $(1-3,6,11)$ give rise to the following signal for a simple carbon-13proton AX spin system,

$$
S_{0}\left(t_{1}, t_{2}\right)=M_{0} \sin \left(2 \pi \delta_{\mathrm{H}} t_{1}\right) \sin \left(\pi J \Delta_{1}\right) \sin \left(\pi J \Delta_{2}\right) \exp \left(-2 \pi i \delta_{\mathrm{c}} t_{2}\right),
$$

TABLE 1

Radiofrequency Phases Usfed in the

Pulse Sequence of Fig 1 on Successive Transients

\begin{tabular}{crrrrr}
\hline $\begin{array}{c}\text { Transient } \\
\text { number }\end{array}$ & $\phi_{1}$ & $\phi_{2}$ & $\phi_{3}$ & $\phi_{\mathbf{4}}$ & $\phi_{\mathbf{R}}$ \\
\hline 1 & 270 & 0 & 0 & 0 & 0 \\
2 & 180 & 0 & 0 & 0 & 90 \\
3 & 90 & 0 & 0 & 0 & 180 \\
4 & 0 & 0 & 0 & 0 & 270 \\
etc. & $\cdot$ & $\cdot$ &. &. & $\cdot$ \\
\hline
\end{tabular}




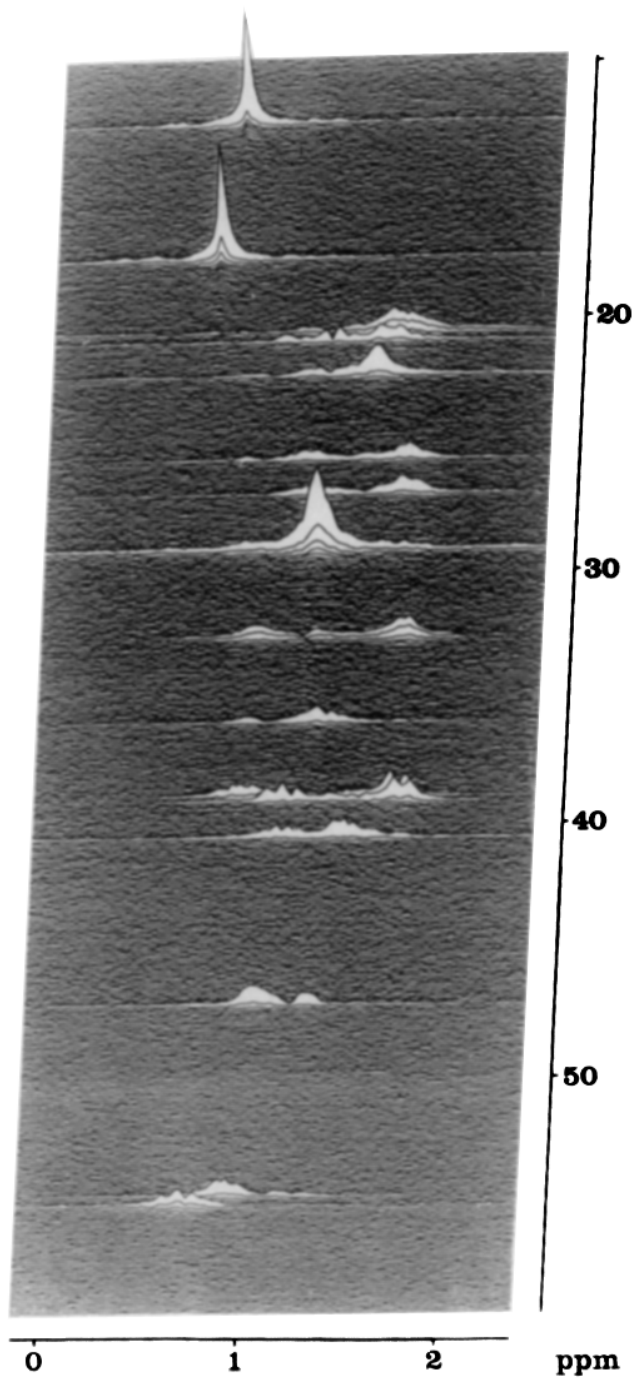

FIG. 2. Two-dimensional chemical shift correlation spectrum of a $1.5 \mathrm{M}$ solution of 5 - $\alpha$-androstane in deuteriochloroform, obtained in approximately 30 min of time averaging using a Varian XL-200 spectrometer operating at $50.3 \mathrm{MHz}$ for carbon-13.

where $J$ is the carbon-proton scalar coupling, and $\delta_{\mathrm{C}}$ and $\delta_{\mathrm{H}}$ are the carbon-13 and proton chemical shifts, respectively. If the phases of the receiver and of the second proton $90^{\circ}$ pulse are now advanced by $90^{\circ}$ in a second experiment, the result is

$$
S_{90}\left(t_{1}, t_{2}\right)=i M_{0} \cos \left(2 \pi \delta_{\mathrm{H}} t_{1}\right) \sin \left(\pi J \Delta_{1}\right) \sin \left(\pi J \Delta_{2}\right) \exp \left(-2 \pi i \delta_{\mathrm{C}} t_{2}\right),
$$

so that summing the two transients restores phase modulation as a function of $t_{1}$,

$$
S_{0+90}\left(t_{1}, t_{2}\right)=2 M_{\mathrm{C}} \exp \left(2 \pi i \delta_{\mathrm{H}} t_{1}\right) \sin \left(\pi J \Delta_{1}\right) \sin \left(\pi J \Delta_{2}\right) \exp \left(-2 \pi i \delta_{\mathrm{C}} t_{2}\right),
$$

allowing discrimination between positive and negative $f_{1}$ frequencies. 

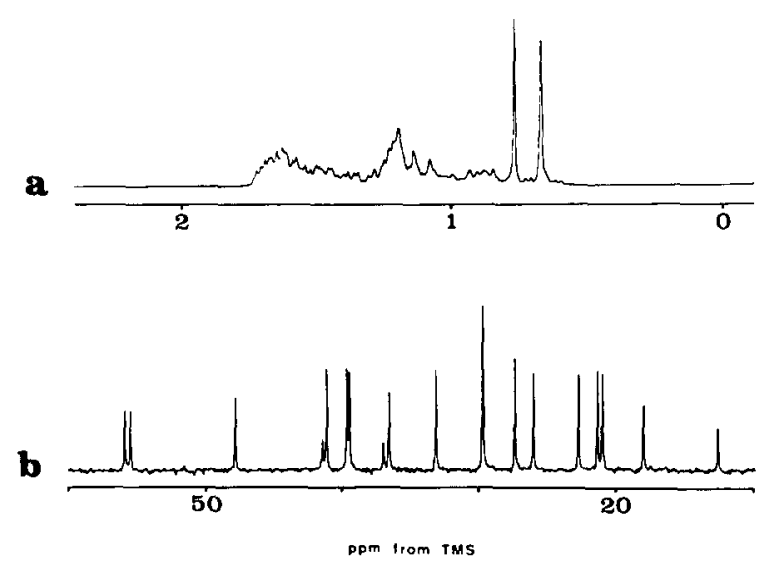

FIG. 3. Conventional proton (a) and carbon-13 (b) spectra for 5- $\alpha$-androstane.

The remainder of the phase cycling scheme of Table 1 serves to suppress residual carbon-13 signals not due to polarization transfer from protons, and to remove the $f_{2}$ zero frequency artifact introduced by imperfect receiver phase balance. Depending on the relative sign of the phase shift introduced between the two proton $90^{\circ}$ pulses, the two exponential terms of $\mathrm{Eq}$. [3] may have the same or, as is the case in [3], opposite senses. The physical significance of choosing opposite senses for the $t_{1}$ and $t_{2}$ precession terms is that this leads to a partial cancellation of static field inhomogeneity effects, giving rise to a coherence transfer echo (17) and thus improving the sensitivity of the experiment slightly. In homonuclear correlation experiments it can be advantageous to select the opposite phase relationship (16), as this can help to discriminate in favor of resonances with natural linewidths.

Figure 2 shows the result of applying the technique described to a solution of the steroid 5- $\alpha$-androstane in deuteriochloroform; for comparison purposes the conventional proton decoupled carbon-13 and proton spectra for this molecule are shown in Fig. 3. Clearly an analysis of the normal proton spectrum would not be feasible even at the highest magnetic fields currently available, whereas the spectrum of Fig. 2 allows all the proton shifts of androstane to be determined using the known carbon-13 assignments (18). Although the method proposed differs only very slightly from that currently in routine use (5), the advantages of reducing decoupler power requirements by a factor of 2 to 4 , reducing the size of data matrix by nearly one-half, and halving the number of $t_{1}$ increments required, enhances significantly the utility of one of the most effective of the many twodimensional NMR techniques now available. Analogous phase cycling schemes are possible in many other two-dimensional NMR experiments, including the important new techniques for mapping chemical exchange and cross-relaxation pathways (19).

\section{ACKNOWLEDGMENTS}

It is a pleasure to acknowledge the advice, encouragement, and stimulation of Ray Freeman, who very generously made available the facilities used in the work described. G.A.M. gratefully acknowl- 
edges discussions with Geoffrey Bodenhausen, who has independently proposed similar experiments. The XL-200 spectrometer was purchased with the aid of an equipment grant from the Science Research Council, while A.B. thanks the Delft University Fund for a stipend.

\section{REFERENCES}

1. A. A. Maudsley And R. R. ERnst, Chem. Phys. Lett. 50, 368 (1977).

2. A. A. Maudsley, L. MUller, and R. R. ERnst, J. Magn. Reson. 28, 463 (1977).

3. G. Bodenhausen and R. Freeman, J. Magn. Reson. 28, 471 (1977).

4. G. Bodenhausen and R. Freeman, J. Am. Chem. Soc. 100, 320 (1978).

5. R. Freeman And G. A. Morris, J. Chem. Soc. Chem. Common., 684 (1978).

6. P. H. Bolton and G. Bodenhausen, J. Am. Chem. Soc. 101, 1080 (1979).

7. L. Müller And R. R. ERnst, Mol. Phys. 38, 909 (1979).

8. G. Bodenhausen, J. Magn. Reson. 39, 175 (1980).

9. R. Freeman and G. A. Morris, Bull. Magn. Reson. 1, 5 (1979).

10. L. D. Hall, G. A. Morris, and S. Sukumar, J. Am. Chem. Soc. 102, 1745 (1980).

11. G. A. Morris and L. D. Hall, J. Am. Chem. Soc., in press.

12. G. A. Morris And L. D. Hall, Can. J. Chem., in press.

13. G. Bodenhausen, R. Freeman, R. Neidermeyer, and D. L. Turner, J. Magn. Reson. 26, 133 (1977).

14. K. Nagayama, K. Wüthrich, and R. R. ERnst, Biochem. Biophys. Res. Commun. 90, 305 (1979).

15. K. Nagayama, A. Kumar, K. WUthrich, and R. R. Ernst, J. Magn. Reson. 40, 321 (1980).

16. A. Bax, R. Freeman, and G. A. Morris, J. Magn. Reson. 42, 164 (1981).

17. A. A. Maudsley, A. Wokaun, and R. R. ERnst, Chem. Phys. Lett. 55, 9 (1978).

18. H. Eggert and C. J. Djerassi, J. Org. Chem. 38, 3788 (1973).

19. S. Macura And R. R. ERnst, Mol. Phys. 41, 95 (1980).

AD BAX*

Gareth A. Morris $\dagger$

Physical Chemistry Laboratory

University of Oxford

South Parks Road

Oxford $O X 13 Q Z$, England

Received November 20, 1980

* Present address: Department of Applied Physics, University of Technology, Delft, The Netherlands.

$\dagger$ Author to whom correspondence should be addressed. 